\title{
Ausblick: Nach der Krise ist nicht vor der Krise
}

\begin{abstract}
„Die Menschheit muss eine Entscheidung treffen. Werden wir den Weg der Uneinigkeit gehen oder den Weg der globalen Solidarität einschlagen? Wenn wir uns für die Uneinigkeit entscheiden, wird dies nicht nur die Krise verlängern, sondern in Zukunft wahrscheinlich noch schlimmere Katastrophen verursachen. Wenn wir uns für globale Solidarität entscheiden, wird dies nicht nur ein Sieg gegen das Coronavirus sein, sondern gegen alle zukünftigen Epidemien und Krisen, die die Menschheit im 21. Jahrhundert heimsuchen könnten [41].“
\end{abstract}

Yuval Noah Harari, NYT-Bestseller-Autor, März 2020

Krisen sind Scheidewege, an denen die Weichen für unsere Zukunft gestellt werden. Ein derart einschneidendes Ereignis hinterlässt Spuren, und so finden wir nach einer Krise zwar in eine Normalität zurück, doch unterscheidet sich diese stets vom gewohnten Alltag vor diesem Ereignis. Diese Auswirkungen lassen sich auf der Ebene des Individuums ebenso beobachten wie in der Art und Weise, wie Unternehmen wirtschaften, Gesellschaften zusammenleben oder politische Systeme funktionieren. Wir sind dieser Entwicklung aber nicht schutzlos ausgeliefert, sondern haben die Chance, diese aktiv mitzugestalten.

Dazu hat beispielsweise das Projekt D2030 erste Antworten in Form von Szenarien für die Frage des „danach“ entwickelt. Hier wurden mehr als 100 Zukunftsexperten aus Wissenschaft, Wirtschaft, Verwaltung, Beratung und Zivilgesellschaft befragt, wie sie die Entwicklung Deutschlands in den kommenden Jahren einschätzen. Auch wenn vereinzelt Sorgen geäußert werden, die bis zu langfristigen Strukturkrisen und der Aushöhlung und Erosion der Demokratie reichen, zeichnet sich insgesamt ein optimistisches Bild. $73 \%$ der Experten (Stand April 2020) rechnen damit, dass die Krise in Deutschland zu mehr Nachhaltigkeit und Gemeinwohl und gleichzeitig zu einer insgesamt 
offeneren Gesellschaft führen wird. Für $97 \%$ der Experten ist dabei klar: Ein „Zurück-zur-alten-Normalität“ wird es nicht geben [42].

Die Ergebnisse zeigen, wie fundamental die Chancen sind, Krisen zu einer positiven Gestaltung der Zukunft zu nutzen. Dies macht Hoffnung, denn wenn wir eines wissen, dann ist es, dass auch in Zukunft Krisen nicht nur unvorhersehbar, sondern auch unvermeidbar bleiben werden. Um Krisen als Teil des (Unternehmens-)lebens begreifen zu können, muss die Fähigkeit etabliert werden, mit Unsicherheit umzugehen. Dazu gilt es, auch die eigene Resilienz zu stärken.

Resilienz bezeichnet dabei die Widerstandsfähigkeit, Krisen zu überwinden und sie durch Rückgriff auf aufgebaute Fertigkeiten als Anlass für Entwicklungen zu nutzen. Dies ist in vielen Bereichen eine sehr nützliche Fähigkeit, wie Prof. Nathan Furr von der INSEAD Universität untersuchte [43]. In seinen Studien beschäftigte er sich mit Personen, die in ihrem Alltag mit großer Unsicherheit umgehen oder besonders resilient sein müssen, wie beispielsweise Gründer, CEOs, Notfallsanitäter, Pokerspieler, Surfer, Startup-Investoren oder Feuerwehrmänner. Dabei konnte er spezifische Muster erkennen, die sehr gut als Leitfaden für resiliente Mitarbeiter, Manager und Unternehmer geeignet sind. Ein wichtiges Muster ist die Fähigkeit, Krisen als Lernmöglichkeit anzusehen. Zudem kann es hilfreich sein, das Leben als Spiel zu betrachten, in dem Frustration und Misserfolg Ansporn zu neuen Höchstleistungen und Optimierungen sind. Häufig ist sogar Dankbarkeit angebracht, da Krisen viele Gelegenheiten dazu bieten, Dinge neu wertzuschätzen. In einigen Fällen wiederum hilft nur die Zufallserkenntnis, dass man eben auch Pech haben kann, und dies kein Grund ist aufzugeben. Besonders inspirierend ist das Heldenmuster, wie es zum Beispiel der australische Filmemacher und ehemalige Notfallsanitäter Benjamin Gilmour beschreibt [43]:

\footnotetext{
„Die meisten Menschen sehen Hindernisse und interpretieren sie als ein Zeichen zum Aufhören. Ich habe gelernt, Hindernisse als Zeichen zu sehen, dass ich in die richtige Richtung gegangen bin. Jede Geschichte, die wir lieben, von Luke Skywalker bis Harry Potter, handelt von dem Helden, der Hindernisse überwindet. Jeder liebt den Helden. Aber die Hindernisse sind es, die den Helden ausmachen. Der einzige Weg, ein Held zu werden ist, die Hindernisse zu überwinden!“
}

Benjamin Gilmour, Regisseur \& Drehbuachautor

Unternehmen können mit den geeigneten Strategien und der richtigen Einstellung Krisen nicht nur überleben, sondern als „Helden“ aus ihnen hervorgehen. Doch ist dies nicht die Zeit der Einzelkämpfer. Wie die Corona-Krise eindrucksvoll beweist, sind unsere Schicksale auf einer globalen Ebene untrennbar miteinander 
verwoben. Niemand steckt alleine in dieser Krise, und niemand kommt alleine wieder aus ihr heraus. Somit liegt es an allen gemeinsam, die Zukunft zum Besseren zu gestalten.

Wir wünschen Ihnen viel Erfolg in dieser und in zukünftigen Krisen! 